\title{
Bilan et perspectives des cultures vivrières dans les pays du Sahel
}

\author{
Johny $\mathrm{EGG}^{1}$ \\ Idrissa WADE ${ }^{2}$ \\ ${ }^{1}$ INRA-SAE2 / UMR MOISA, \\ 2 place Viala, \\ 34060 Montpellier Cedex 1 \\ <egg@ensam.inra.fr> \\ 2 doctorant Agro-M/UMR MOISA, \\ 2 place Viala, \\ 34060 Montpellier Cedex 1 \\ <wade@ensam.inra.fr>
}

\begin{abstract}
Twenty-five years after the beggining of the policies of adjustment and liberalization which is the situation of the food crops in the countries of the Sahel? The balance-sheet is approached by contrasting the cereals sub sector, facing to a very strong control by the State, and the onion whose growth is related to the increase in the demand of the great urban centers. The increase in the production of cereals was accompanied by deep changes in the structures. The market became more efficient and better integrated on a regional scale. But, the level and the instability of the prices of cereals increased, making difficult the provisioning of the poor. Clear differences in trajectories appear. Mali, which implemented a policy of reforming cereals market and successful the revival of rice sector, must manage at the same time the improvement of the competitiveness of its cereals and the access to cereals of the low incomes consumers. Niger must face the increase in the vulnerability of a great part of the rural households. The production of onion experienced a spectacular increase in Senegal after the devaluation of franc FCA in response to the urban market. That of Niger intended mainly for the close countries progressed more slowly. But in both cases, the regulation of the European onion imports constitutes a paramount stake.
\end{abstract}

Key words: liberalisation, onion, cereals, Sahel

ves des cultures vivrières dans les pays $d u$ Sahel ?

Dans les bilans des réformes des marchés produits agricoles des pays les moins avancés (PMA), un consensus s'est établi depuis quelques années dans la littérature pour souligner qu'en Afrique subsaharienne les réformes sont loin d'avoir atteint les résultats attendus [1]. Les taux de croissance du secteur agricole sont faibles comparés à ceux des PMA d'Amérique latine et d'Asie, la pauvreté s'aggrave dans de nombreux pays, la malnutrition et les crises alimentaires perdurent.

Au-delà de ce constat global, apparaît une image plus nuancée, avec une diversité de situations traduisant des différences de trajectoires.

Le présent article cherche à préciser cette image dans le cas des pays du Sahel. Comme dans la plupart des PMA, l'environnement économique et institutionnel de la production et distribution de denrées alimentaire a subi dans les pays sahéliens de grands changements depuis le milieu des années 1980 sous les effets conjugués des politiques économiques de libéralisation (ajustement structurel, libéralisation des marchés agricoles nationaux et internationaux), des crises économiques et financières, de la dégradation de la fertilité des sols et de la croissance démographique et de l'urbanisation.

Vingt-cinq ans après le lancement des politiques d'ajustement structurel et de stabilisation des économies, au moment où le financement de l'agriculture revient à l'ordre du jour dans les politiques d'aide, comme une nécessité pour atteindre les Objectifs du Millénaire du Développement (OMD) de réduction de la pauvreté, quelles sont la situation et les perspecti-
On abordera ce bilan en contrastant deux secteurs qui jouent un rôle essentiel dans I'alimentation des populations, mais qui ont fait l'objet $d^{\prime}$ un traitement très différent par les politiques économiques: les céréales et les produits maraîchers, l'oignon en particulier. Les filières des céréales, base du régime alimentaire sahélien, ont été soumises dès les années 70 à un encadrement très fort au nom des politiques d'autosuffisance alimentaire. À l'image des cultures d'exportation comme le coton, elles ont été au cœur du débat sur la libéralisation, en particulier la filière riz. Les cultures maraîchères n'ont pas reçu la même attention des politiques agricoles : en dehors des projets de développement, leur croissance est davantage liée à l'augmentation de la demande des grands centres urbains.

Pour chacun de ces secteurs, on contrastera différentes trajectoires. Pour les céréales, on opposera les cas du Mali, qui est devenu exportateur sur le marché régional, et le Niger, dont la sécurité alimentaire reste très fragile. Pour I'oignon, on soulignera les cas du Niger où la production est principalement destinée à l'exportation vers les pays voisins et celui du Sénégal où la production doit répondre à la demande urbaine croissante.

On commencera par rappeler le rôle des céréales et des produits maraîchers dans l'économie des pays sahéliens et les grands traits des politiques de libéralisation mises en œuvre. Ensuite, on examinera l'évolution de ces cultures vivrières en soulignant les changements dans les structures de production et distribu- tion. En conclusion, on soulignera les principaux enjeux.

\section{Le rôle central des céréales dans les économies des pays sahéliens}

Le Niger et le Mali sont classés parmi les pays les plus pauvres de la planète (au dernier rang pour le Niger selon I'indicateur IDH du PNUD). A l'échelle du Sahel, ils figurent néanmoins comme des "grands" pays agricoles. En dehors de l'exploitation de modestes richesses minières et des revenus de l'émigration, l'essentiel des ressources repose sur l'agriculture et l'élevage.

Les céréales occupent une place prépondérante dans l'économie des deux pays. Elles assurent plus de $69 \%$ des calories du régime alimentaire moyen au Mali et $72 \%$ au Niger, et constituent le pilier des systèmes de production-consommation [3]. Les céréales sèches (mil, sorgho et maïs) sont cultivées en majorité dans des systèmes à faible productivité par de petits producteurs peu insérés dans le marché, sauf au Mali dans les zones cotonnières du sud qui connaissent une certaine intensification. Soumise aux aléas de la pluviométrie, la production de céréales sèches est caractérisée par de fortes fluctuations dans le temps (variations interannuelles) et aussi dans l'espace. Par ailleurs, en raison de la part relativement faible de la production qui est commercialisée par les producteurs (estimée en général à 15-20\%), le marché (au sens du volume de céréales soumis à transaction marchande) est étroit. De ce fait il est caractérisé 
par une forte volatilité des prix, temporelle et spatiale, qui renforce les stratégies d'aversion au risque des producteurs et des opérateurs commerciaux.

\section{Le rôle croissant des cultures maraîchères}

Le secteur maraîcher au Sénégal a connu un développement rapide ces dernières années. La production des principales spéculations maraîchères a doublé en l'espace de 10 ans, passant de 150000 tonnes en 1992 à plus 300000 tonnes en 2004 [2]. Cette évolution s'est déroulée dans un environnement marqué par l'augmentation de la demande due à I'urbanisation et par la dévaluation du franc CFA qui a rendu la production locale plus attractive. L'oignon occupe le premier rang des cultures maraîchères avec une superficie de près de 4900 ha en 2002 et une production d'environ 90000 tonnes [2]. Sa consommation est passée de $6 \mathrm{~kg} /$ pers/an en 1990 à $13 \mathrm{~kg} / \mathrm{pers} / \mathrm{an}$ en 2003 [3]. L'oignon est le principal légume consommé et représente $20 \%$ des dépenses totales en légumes [4]. Les villes constituent les plus gros centres de consommations de légumes.

Contrairement au Sénégal où la production d'oignon est principalement destinée à la satisfaction de la demande locale, au Niger l'oignon est le troisième produit $d^{\prime}$ exportation en valeur après I'uranium et le bétail (avec un apport en devises de près de 15 millions de dollars US par an entre 1995 et 2003 [3]). Dans un contexte où les conditions climatiques défavorables exposent les petits producteurs à des difficultés alimentaires, les revenus issus de ces cultures sont essentiels, notamment pour l'achat de céréales.

\section{Un processus de libéralisation étendu dans le temps et contrasté}

\section{La libéralisation du marché céréalier}

À l'image des autres pays sahéliens, la libéralisation des filières céréalières s'est déroulée au Mali et au Niger sur près de 15 ans, en suivant un "agenda» similaire: suppression $d u$ monopole des Offices de commercialisation (au Mali dès 1982), libéralisation des prix des céréales sèches en 1986 et 1987, suivie de celle des prix du riz, tergiversations durant une dizaine d'années dans la libéralisation des importations de riz (effective en 1990 au Mali et en 94 au Niger), la dévaluation du FCFA de 1994 venant clore la phase de libéralisation et d'ajustement.

Alors que le Niger éprouvait des difficultés à redéfinir sa politique agricole (il définira mieux, mais plus tard, une politique de prévention et gestion des crises alimentaires), le Mali mettait en place dès 1981, avec les bailleurs de fonds, un dispositif original d'accompagnement des réformes, le PRMC (Programme de restructuration du marché céréalier).

Le secteur agricole ayant été géré jusque-là de façon administrée à travers le monopole public des offices de commercialisation et les prix fixés au producteur et au consommateur au même niveau sur tout le territoire, on pouvait s'attendre à des répercussions fortes de la politique de libéralisation sur la sécurité alimentaire. Dans ce contexte de modification radicale des règles du jeu, le PRMC a fait des efforts importants pour construire les institutions nécessaires au fonctionnement du marché : mise en place d'un SIM (systèmes d'information du marché céréalier), appui à la constitution de GIE de commerçants, programme de crédit aux producteurs (via les $A V$ - associations villageoises et les OP - organisations de producteurs), mise en place d'une politique de prévention des crises avec un SAP (système d'alerte précoce) $[5,6]$.

\section{L'évolution des cultures céréalières au Mali et au Niger}

\section{Une croissance de l'offre qui ne garantit pas la sécurité alimentaire}

La production totale de céréales a connu une forte augmentation dans tous les pays du Sahel, à l'exception notable du Sénégal où elle a baissé. Pour autant, les variations interannuelles de la production céréalière se maintiennent. En 25 ans, la production de céréales a presque triplé au Mali, passant de 1,1 million de tonnes au début des années 80 à 2,7 millions de ton-

Tableau 1. Évolution de la composition de la production céréalière au Mali.

\begin{tabular}{|lcc|}
\hline & $\mathbf{1 9 8 0 - 8 2}$ & $\mathbf{2 0 0 2 - 0 4}$ \\
\hline Mil-Sorgho \% & 80,4 & 54,3 \\
Maïs \% & 4,5 & 13,6 \\
Riz paddy \% & 12,2 & 31,4 \\
Total céréales 1000 t & 1148,9 & 2682,8 \\
\hline
\end{tabular}

Source : FAOSTAT.

Tableau 2. Importations de céréales en volume au Mali et Niger (1 000 t).

\begin{tabular}{|lcccc|}
\hline & \multicolumn{2}{c}{ Riz } & \multicolumn{2}{c|}{ Blé } \\
\cline { 2 - 5 } & $\mathbf{1 9 8 0 - 8 2}$ & $\mathbf{2 0 0 2 - 0 4}$ & $\mathbf{1 9 8 0 - 8 2}$ & $\mathbf{2 0 0 2 - 0 4}$ \\
\hline Mali & 50052 & 40708 & 18481 & 32873 \\
Niger & 43900 & 126125 & 8158 & 7944 \\
\hline
\end{tabular}

Source : FAOSTAT. nes en 2002-2004. Cette croissance ne doit pas au plus de retrouver le niveau de production par habitant du début des années 60 au Mali, et au Niger ce ratio diminue.

Au Mali, l'augmentation de la production s'est accompagnée d'une profonde modification de la composition de l'offre de céréales locales, les cultures traditionnelles - mil et sorgho cédant de la place au riz et au maïs (tableau 1). Les mil-sorgho ne représentent plus que $54 \%$ de la production totale de céréales (contre $80 \%$ au début des années 1980), alors que dans le même temps la part du maïs, négligeable il y a 25 ans, atteint près de $14 \%$ [7] et celle du riz paddy plus de $30 \%$.

Au Niger, le mil et le sorgho restent encore largement les céréales dominantes $(97 \%$ de la production totale) avec toutefois des parts relatives qui évoluent, le sorgho diminuant au profit du mil.

L'augmentation de la production de mil et sorgho résulte essentiellement d'un accroissement des surfaces emblavées, les rendements ayant tendance à stagner, à baisser au Niger. Par contre, les rendements du maïs ont nettement progressé au Mali (suite à l'intensification dans les zones cotonnières) et ceux du riz davantage encore.

Les importations totales de céréales ont augmenté dans tous les pays du Sahel. L'augmentation est due aux importations de blé et, surtout, de riz dont les volumes importés ont crû dans une forte proportion sauf au Mali où les importations de riz ont baissé jusqu'à récemment (elles sont élevées depuis deux ans). Contrairement à certaines prévisions, les importations de riz et de blé n'ont pas été freinées par la dévaluation du franc CFA ; elles augmentent même depuis le réajustement monétaire (tableau 2). faire illusion : les bons résultats permettent tout 


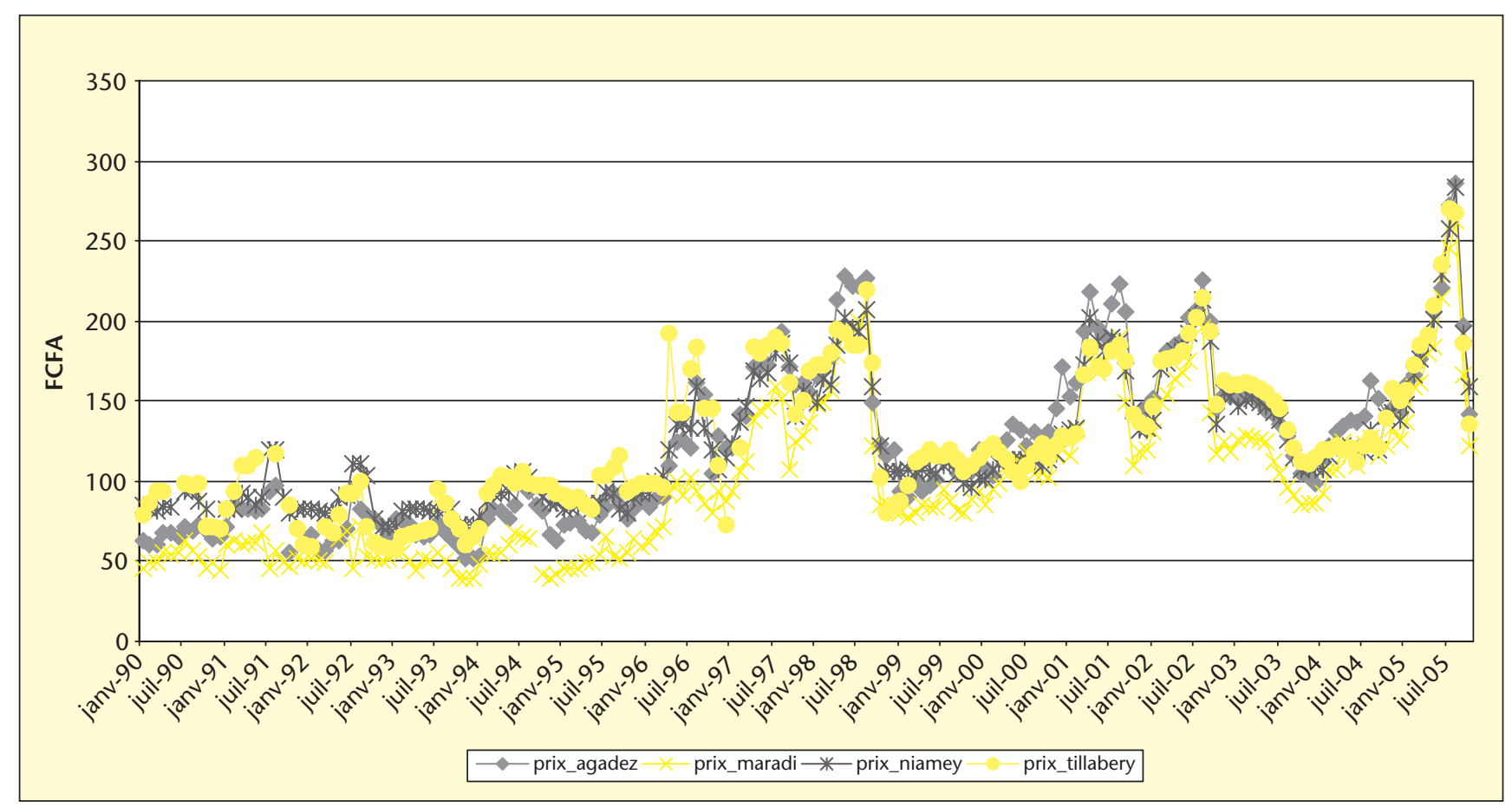

Figure 1. Prix nominal du mil sur les marchés du Niger 1990-2005. Source: SIMA.

L'aide alimentaire ne représente plus qu'une part marginale des importations de céréales au Mali, par contre au Niger elle contribue à l'approvisionnement des ménages à hauteur de $5 \mathrm{~kg} / \mathrm{hab} / \mathrm{an}$ en moyenne sur la période 1987/88-2005, pour une consommation apparente moyenne de $215 \mathrm{~kg} / \mathrm{hab}$. [8].

En dehors du Niger devenu un importateur structurel du Nigeria dans les années 80 déjà, le commerce régional de céréales portait en grande partie la réexportation. La dévaluation a eu pour effet de stopper ces activités et de relancer le commerce des productions locales. Le Mali est devenu ces dernières années un fournisseur important de ses voisins en céréales sèches: Sénégal - en grande partie pour l'alimentation des volailles - Côte d'Ivoire, Burkina, et le Niger lorsque les conditions d'offre du Nigeria sont moins compétitives [5, 9].

\section{La forte augmentation des prix des céréales}

Le trait marquant de l'évolution des prix à la consommation est le trend à la hausse qui s'est enclenché suite à la dévaluation du franc CFA $[10-12,5,13]$. Du milieu des années 80 au milieu des années 90 , les prix des céréales avaient relativement baissé dans l'ensemble des pays du Sahel. Après la dévaluation du franc CFA, ils franchissent un palier ; le trend de hausse se poursuit dans la période récente, mais à travers de fortes fluctuations interannuelles, comme l'illustre l'évolution des prix du mil sur les marchés (de regroupement et des zones déficitaires) du Niger (figure 1).

\section{Les changements dans les structures du marché céréalier}

\section{L'insertion des producteurs au marché reste faible}

Une des hypothèses fortes des politiques de réforme des marchés céréaliers était que la libéralisation entraînerait une hausse durable des prix qui inciterait les producteurs à augmenter la production et la part commercialisée des céréales. Or si la hausse des prix s'est effectivement produite, l'élasticité de la production est restée globalement faible. La réponse aux incitations de prix est en effet concentrée sur certaines zones (au Mali, la zone cotonnière et I'Office du Niger) et un petit nombre de producteurs.

Ainsi, la sécurité alimentaire reste-t-elle la préoccupation essentielle de la grande majorité des ménages ruraux. De nombreux travaux ont expliqué les raisons de ces comportements tournés vers la sécurisation de la consommation et des revenus de la famille. Ils mettent en avant les risques élevés liés à la production dans un environnement d'aléas climatiques et de marchés imparfaits (manque d'information, absence de marché du crédit, précarité des réseaux de transport, difficultés d'approvision- nement en intrants). Dans ce contexte, l'objectif de sécurisation des ressources induit une préférence pour les cultures aux rendements plus faibles mais aux résultats plus sûrs (résistance aux aléas) et des comportements d'assurance contre le risque qui ne permettent pas l'investissement.

La grande majorité des producteurs des pays du Sahel sont des acheteurs nets de céréales sur le marché : ils vendent après la récolte pour répondre à leurs besoins monétaires, mais doivent acheter plus tard des céréales pour leurs besoins de consommation. Pour les acheteurs nets de céréales, la tendance à la hausse des prix des céréales de ces dernières années et l'accroissement de leur instabilité, ne constituent pas une opportunité, mais bien un obstacle supplémentaire à l'intensification de la production [9].

\section{Les effets d'entraînement du coton sur le mais}

La complémentarité entre la culture du coton et celle des céréales s'est vérifiée au long de la période. La production céréalière a bénéficié d'externalités positives en association avec la culture du coton encadrée par les sociétés cotonnières dans les pays de la zone franc. Le maïs en a été le principal bénéficiaire, notamment au Mali où l'essor de la production de maïs est concentré dans la zone CMDT de Mali-Sud. Le coton permet l'accès aux intrants (indispensable à la réalisation du potentiel du 
maiis), au crédit et à l'équipement, qui créent les conditions pour l'augmentation des rendements en céréales. Ensuite, le coton permet une sécurisation du revenu monétaire qui crée les conditions d'un comportement « commercial » du producteur de céréales et lui permet d'écouler sa production plus tard dans la saison, à de meilleurs prix.

\section{Le succès de la libéralisation de la filière riz au Mali}

Les performances des filières riz au Sahel sont inégales suivant les pays. Au Mali, dans la zone de l'Office du Niger, où la production est entièrement dégagée de l'aléa climatique du fait d'un important (et ancien) investissement public dans les aménagements hydroagricoles, la libéralisation fait figure de success story [14]. Elle a eu un effet positif manifeste sur la production de riz parce qu'elle s'est combinée avec d'autres facteurs (réaménagement des parcelles, diffusion d'innovations techniques comme le repiquage, organisation des producteurs, etc.). L'intensification de la production résulte ici d'un triple changement : les réformes de politique économique (libéralisation et dévaluation), les innovations techniques et les changements organisationnels $[15,16]$.

\section{Un pouvoir de négociation accru des producteurs}

L'évolution des rapports de prix en faveur des producteurs traduit un renforcement de leur pouvoir de négociation avec les commerçants. Celui-ci résulte de plusieurs facteurs, dont I'amélioration progressive de l'information, l'augmentation du nombre d'intervenants sur le marché (qui se font concurrence en cas de pénurie), I'organisation des producteurs en groupements.

Ce changement de rapport de force ne concerne pas la majorité des producteurs, mais depuis la fin des années 90 , il est suffisant pour tirer le niveau des prix des céréales à la hausse lorsque l'offre n'est pas très abondante [5].

\section{Un marché plus efficace mais qui reste incomplet}

Les études soulignent que le marché est devenu plus concurrentiel et que son efficacité s'est améliorée. Ce diagnostic s'appuie sur des analyses des prix du SIM et sur des enquêtes auprès des commerçants [9]. Ces résultats indiquent une amélioration de la circulation de l'information, de la concurrence entre opérateurs et des transports et signifient que l'accès des zones déficitaires au marché est facilité. Au Mali, la concurrence a augmenté en premier lieu dans la commercialisation du riz, qui était contrôlée par quelques importateurs dans les années 80 , et qui a vu l'entrée de nombreux intervenants suite à la libéralisation et à la multiplication des décortiqueuses privées. La commercialisation des céréales sèches, moins concentrée au départ, a connu la même évolution au Mali, Burkina et Niger. Les formes $\mathrm{d}^{\prime}$ organisation traditionnelle en réseau que privilégient les commerçants dans les pays du Sahel face au risque perdurent, mais les réseaux se sont ouverts ou, comme dans le cas du Niger, ils ont éclatés, les commerçants du Nigeria venant traiter eux-mêmes leurs affaires au Niger [10].

Les difficultés à mettre en place un système de crédit à la production suite au retrait de l'État et à l'échec des grandes banques constituent une contrainte majeure dans le marché libéralisé. Dans la zone de l'Office du Niger au Mali, un système décentralisé de caisses villageoises de crédit permet l'accès au crédit à la majeure partie des producteurs, mais se limite à l'approvisionnement en intrants pour la riziculture [17].

\section{Un marché intégré à l'échelle régionale}

La dévaluation du FCFA de début 1994 a entraîné des changements importants dans le marché céréalier. D'une part les coûts de transfert ont nettement augmenté, d'autre part la dévaluation a stimulé les exportations de céréales sèches du Mali vers les pays limitrophes. Aujourd'hui, le marché des céréales peut être considéré comme intégré à l'échelle régionale ouest-africaine.

Pour le Mali, cela signifie que les prix au consommateur ont été tirés vers le haut. Avant la dévaluation, les prix à la consommation ont varié principalement en fonction des fluctuations de la production. À partir de 1995, une certaine déconnexion des prix apparaît : l'évolution des prix intérieurs ne dépend plus de la seule production, mais également de la demande des centres urbains et des pays voisins. Le marché est tiré par la demande.

\section{L'instabilité des prix demeure une contrainte forte}

Les gains en performance du marché céréalier se sont accompagnés d'une plus grande instabilité des prix à la production et à la consommation.

Cette instabilité des prix a plusieurs effets négatifs. En premier lieu, elle affecte les consommateurs les plus pauvres, urbains et ruraux, qui consacrent une proportion de leur revenu plus importante que les autres à l'achat des céréales. Ensuite, elle représente un facteur de risque pour les producteurs, notamment de maïs, et pour les opérateurs du stockage et de la transformation artisanale ou industrielle. Dans ce sens, elle constitue une sérieuse limite à l'impact à moyen et long terme de la réforme, car elle entretient le cercle vicieux d'un marché étroit en n'incitant pas les producteurs à augmenter les volumes mis en marché et les commerçants à augmenter les stocks stabilisateurs.

\section{La prépondérance des céréales se maintient dans la consommation}

Dans le contexte de réduction du pouvoir d'achat, la part consacrée aux céréales et la consommation moyenne de céréales ont augmenté au Mali après la dévaluation. Le riz a pris une part importante dans cette évolution, les consommateurs aux revenus les plus élevés ayant presque doublé leur consommation et diminué celle de céréales sèches, alors que les consommateurs à bas revenu ont augmenté leur consommation de mil-sorgho sans réduire celle de riz [18-20].

De 1989 à 2001, la consommation moyenne par habitant a augmenté au Mali de $7 \%$ en riz et de $27 \%$ en mil, mais diminué de près de $33 \%$ pour le sorgho et de $13 \%$ pour le maïs (selon les enquêtes de la DNSI) [21].

\section{De nouvelles formes d'insécurité alimentaire}

Le Mali a retrouvé un niveau moyen de sécurité alimentaire. Dans les zones déficitaires, la sécurité alimentaire s'est améliorée du fait d'une disponibilité accrue et plus stable des céréales sur les marchés et d'une diversification croissante des sources de revenus des populations. Au Niger, par contre, l'insécurité alimentaire concerne une large part de la population et la crise alimentaire de 2005 a mis en évidence la dégradation des stratégies d'atténuation des crises des populations vulnérables. La production agricole des familles très vulnérables ne couvre plus que quelques mois de consommation et l'émigration dans les pays voisins prend une place prépondérante dans les stratégies. La consommation passant davantage par le marché, ces ménages ont subi de plein fouet la flambée des prix en 2005 [22].

Au-delà, l'insécurité alimentaire prend de nouvelles dimensions dans les deux pays, comme ailleurs au Sahel, avec :

- une malnutrition chronique et aiguë des enfants de moins de 5 ans, y compris dans les zones qui dégagent des excédents céréaliers $[23,24]$;

- une baisse du pouvoir d'achat des catégories socio-professionnelles aux revenus fixes (fonctionnaires, salariés) qui s'est accentuée depuis la dévaluation, entraînant une détérioration du niveau et de la qualité de la consommation alimentaire ;

- une détérioration de la situation alimentaire des pauvres urbains qui doivent faire face à la fois à la hausse des prix des céréales (qui représentent une part essentielle de leurs dépenses) et à la réduction des «filets de sécurité " liés aux différentes formes de solidarité familiale. 


\section{L'évolution de la culture de l'oignon}

Les réformes prises dans le cas des politiques d'ajustement structurel ont peu touché la production maraîchère dans la mesure où celle-ci ne faisait pas l'objet de politiques de fixation de prix, ni de la mise en place de structures particulières chargées de la commercialisation. De ce fait, il n'y a pas eu de changements de structure suite à la libéralisation mais une structuration du secteur maraîcher par les opérateurs eux-mêmes pour répondre à une forte demande.

\section{La dynamique de la production}

Au Niger, la culture d'oignon s'est développée suite aux sécheresses de 1973 et 1984 qui ont accentué le passage d'un système de jardinage destiné à l'autoconsommation à la production maraîchère de contre-saison cultivée pour les revenus. Ce passage a pu se faire grâce à I'appui de projets de développement agricole et de I'amélioration du transport. Des projets tels que le Projet Basse vallée de la Tarka, le Projet Intégré Keita, le Programme Spécial FIDA, le PDRT Tahoua, ont permis la mobilisation d'importants moyens pour des forages de puits, des équipements en motopompes permettant ainsi à la culture d'oignon de se développer dans le département de Tahoua. L'essentiel de la production d'oignon du Niger est réalisé dans les départements de Tillabéry, de Dosso, de Tahoua ( $80 \%$ de la production totale), de Maradi et de Zinder dans le sud du pays sur des périmètres irrigués, dans la vallée du fleuve Niger et le long des cours d'eau tel que le Goulbi dans le département de Maradi. Cette production est essentiellement destinée à l'exportation. En effet, avec une demande locale inférieure à $10 \%$ de la production, des pertes évaluées à $30 \%$, des besoins pour l'autoconsommation et les semences à $10 \%$ [25], le disponible pour l'exportation est supérieur à $50 \%$ de la production. L'exportation $s^{\prime}$ effectue principalement vers la Côte d'Ivoire $(35,8 \%)$, le Nigeria $(33,5 \%)$ et le Chana $(23,9 \%)$ [26]. La commercialisation est effectuée par des commerçants privés du secteur informel, le plus souvent organisés en réseaux [27].

Au Sénégal, une forte croissance de la filière oignon est à noter suite à la dévaluation ayant entraîné la hausse des prix de l'oignon importé. $C^{\prime}$ est ainsi que la production d'oignon essentiellement assurée par la région des Niayes s'est étendue à la vallée du fleuve Sénégal. Les surfaces en oignon n'y excédaient pas 500 ha avant 1994 [28] elles dépassent 3000 ha depuis 2003. Le rôle central de l'oignon dans les revenus monétaires des exploitations de moyenne vallée est pour beaucoup dans ce développement étant donné la faible rentabilité du riz et ses difficultés d'écoulement. La production d'oignon est essentiellement destinée à la consommation nationale et acheminée vers les grands centres de consommation [29, 30]. Cependant, elle ne couvre pas I'intégralité de la demande du fait de la saisonnalité des récoltes et de la qualité du produit qui ne permet pas une longue conservation. La filière oignon a pu bénéficier de projets de développement comme le Projet d'appui à l'entreprenariat paysan (PAEP) œuvrant pour la promotion de la compétitivité des filières oignon et pommes de terre sur les marchés domestiques et sous régionaux et le Projet de promotion des petites et moyennes entreprises horticoles (PPMEH) avec un fort volet microcrédit.

\section{La concurrence de l'oignon importé}

Les prix de l'oignon sont également influencés par la présence de l'oignon importé. En effet, au-delà de se positionner uniquement de façon complémentaire à la production locale, les importateurs ont développé au cours des dernières années des stratégies de conquête du marché. C'est ce qu'illustre le rapport production/ importation (figure 2). Si jusqu'en 1997 ce rapport suit une tendance à la baisse, à partir de 1998 une hausse régulière est observée.

Le calendrier de l'entrée des importations pose problème à l'écoulement de l'oignon local. Les importations démarrent en général dès le mois de juillet - les producteurs hollandais cherchant à se débarrasser des reliquats de stocks avant l'arrivée de la nouvelle récolte - et se poursuivent durant les deux ou trois premiers mois de l'année, alors que les premiers oignons locaux arrivent en février [31].

La régulation des importations a été un problème récurrent pour le développement de
I'oignon local. Jusqu'en 1995, les importations étaient soumises à des mesures de contingentement qui imposaient aux importateurs de commercialiser de l'oignon local à raison du tiers des volumes importés [32]. Cette mesure de restriction quantitative a été supprimée en décembre 1995 et les droits d'entrée ont été abaissés de 5 points, pour s'élever à $50,8 \%$ du prix CAF [28]. Depuis 2000, I'entrée en vigueur du tarif extérieur commun de I'UEMOA a conduit à une baisse du taux de prélèvements à I'importation à 26,5\% (auxquels s'ajoutent $18 \%$ de TVA).

Pour faire face à la forte hausse des volumes importés et à la persistance des importations en période de pleine récolte, des restrictions sur les importations entre juillet et fin septembre ont été prises en 2004 à l'initiative de l'ARM (Agence de régulation des marchés) et en concertation avec les organisations de producteurs et de commerçants. Ces restrictions quantitatives constituent une avancée significative mais leur efficacité réelle sur les volumes importés reste à confirmer. La protection du marché intérieur, durant les périodes de pleine commercialisation de l'oignon local apparaît nécessaire. Mais elle nécessite une très bonne connaissance des stratégies des importateurs et un travail conjoint entre l'État et les organisations de producteurs et de commerçants.

L'enjeu majeur pour les producteurs sénégalais est de parvenir à gagner des parts de marché sur les importations. Pour cela, I'amélioration de la qualité, telle qu'elle a été initiée avec la création de l'oignon de marque "soblé Niayes", soutenue par le PAEP, est nécessaire. Le développement d'infrastructures de stockage est crucial. II s'est limité dans la vallée à quelques unités financées par le projet FEDPME dans les années 90 , mais délaissées au

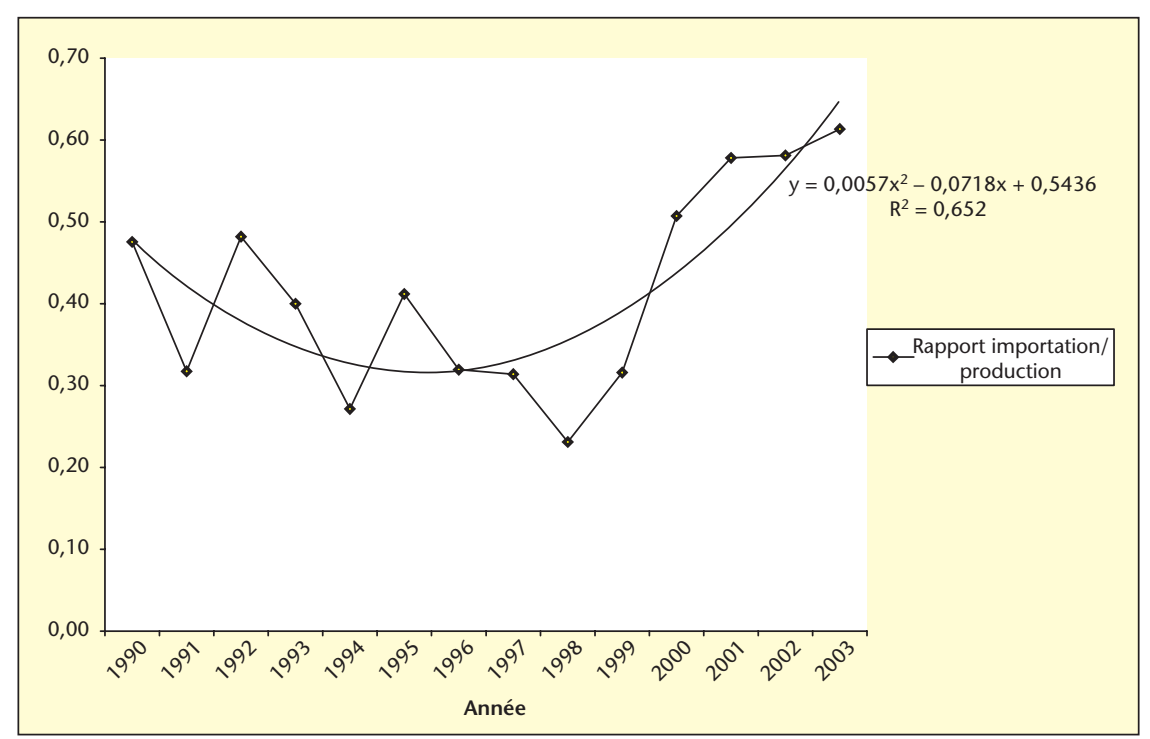

Figure 2. Évolution du rapport importation/production de 1990 à 2003. 
bout de quelques années, essentiellement pour des problèmes de gestion collective. Les unités de stockage mises en place avec l'appui du PAEP dans les Niayes, entre 1999 et 2000, semblent donner des résultats plus satisfaisants, en particulier celles gérées par des entrepreneurs privés [31].

Vis-à-vis de l'oignon du Niger, la concurrence des importations européennes s'exerce sur le marché régional. L'impact des APE (accords de partenariat économique avec I'Union européenne) pourrait être important pour le Niger dont les exportations reposent en grande partie sur les productions agropastorales vendues au Nigeria et dans les pays du golfe de Guinée (Ghana, Côte d'Ivoire notamment). Une libéralisation des exportations pourrait entraîner des effets d'éviction des exportations nigériennes des marchés côtiers, comme ce fut déjà le cas avec les exportations de viande bovine ou de volailles européennes. Aussi, la création d'une véritable union douanière au niveau de I'espace CEDEAO et la fluidification des marchés intrarégionaux est un enjeu important pour les productions maraîchères du Niger [26].

\section{Conclusion}

L'analyse fait apparaître des différences notables de trajectoires, à l'image du constat plus général d'évolution des PMA.

Au Mali, qui a mis en œuvre une politique d'accompagnement de la libéralisation, les filières céréalières deviennent plus compétitives, mais l'instabilité et le niveau élevé des prix pénalisent les groupes de population à bas revenu. L'enjeu auquel la politique céréalière doit faire face est d'améliorer à la fois la compétitivité des céréales sur les marchés intérieur et régional et l'accès des consommateurs à bas revenus qui consacrent une part majeure de leurs budgets aux céréales. La promotion de la qualité des céréales sur le marché régional peut constituer une voie dans ce sens : la segmentation des marchés permettant de mieux valoriser les céréales de qualité sans stimuler la hausse des prix des autres céréales [33].

Au Niger, qui doit faire face à l'augmentation de la vulnérabilité d'une partie importante des ménages ruraux, l'enjeu de la sécurité alimentaire apparaît prioritaire. Avec un taux de croissance de la population qui est un des plus élevé du monde, l'intensification des cultures vivrières devient une question primordiale.

Les productions maraîchères, I'oignon en tête, ont connu un développement spectaculaire au Sénégal après la dévaluation du franc FCA en réponse à l'augmentation de la demande urbaine. Au Niger, la production d'oignon destinée principalement à l'exportation sur le marché régional continue à jouer un rôle important pour la balance des paiements et la sécurité alimentaire des producteurs. Dans les deux cas, la régulation de la concurrence des importations d'oignon d'Europe (sur le marché du Sénégal ou sur les marchés régionaux clients du Niger) est un enjeu primordial pour le développement des filières.

\section{RÉFÉRENCES}

1. KHERALLAH M, DELGADO C, GABRE-MADHIN E, MINOT N, JOHNSON M. Reforming Agricultural Markets in Africa. IFPRI, John Hopkins University Press, 2002.

2. DIRECTION DE L'HORTICULTURE. Statistiques Horticoles. 2004.

3. FAOSTAT.

4. DIRECTION DE LA PREVISION ET DES STATISTIQUES. Enquêtes Sénégalaises sur les ménages. 2004.

5. EGG I. Etude de l'impact de la libéralisation sur le fonctionnement des filières céréalières au Mali. Rapport de synthèse. Bamako : PRMC, 1999.

6. DEMBELE N, STAATZ J. The impact of Reform on Agricultural Transformation in Mali. MSU, Staf Paper 1999 : 29.

7. BOUGHTON D, REARDON T. Will promotion of coarse grain processing turn the tide for traditional cereals in the Sahel? Recent empirical evidence from Mali. Food Policy 1997; 22(4) : 307-16.

8. PAM/INTERFAIS. Moniteur de I'aide alimentaire. Aide alimentaire aux pays membres du CILSS 2004-2005. Rome, décembre. 2005.

9. DEMBELE N, STAATZ J. Response of Cereals Traders to Agricultural market Reform in Mali. In : Bingen J, Robinsons D, Staatz J, eds. Democracy and Development in Mali. East Lansing: MSU Press, 2000 : 145-65.

10. HAMADOU S. La hausse des prix des céréales au Niger. Club du Sahel/ REDES, 1999.

11. KI-ZERBO B. La hausse des prix des céréales au Burkina Faso: constats et analyse des changements institutionnels. Club du Sahel/OCDE, 1998.

12. REDES. La hausse des prix des céréales au Niger. Conséquences sur la sécurité alimentaire des populations. Synthèse. Club du Sahel/CILSS, 1999.

13. TRAORE K, JEUDY E, BLEIN R. Analyse des déterminants de la hausse des prix des céréales au Burkina, Mali et Niger à l'issue de la campagne agricole 2001/2002. 2003.

14. KUPER M, TONNEAU J-P. L'Office du Niger, grenier à riz du Mali. Ed. Karthala et CIRAD, 2002.

15. CHOUIN-KUPER A, MARIKO D, KELLY V. La filière riz à l'Office du Niger au Mali : une nouvelle dynamique depuis la dévaluation du franc CFA. IER/INSAH/MSU, 1999.
16. EGG J, DEME M. La libéralisation du marché du riz au Mali : un succès combinant progrès technique, politique économique et organisation des acteurs. In : Kuper M, Tonneau JP, eds. L'Office du Niger, grenier à riz du Mali. Karthala et CIRAD, $2002: 88-92$.

17. MEGANK M. Unravelling the institutional knot: the role of institutions and coordination in commodity sector performance : the case of irrigated rice in Mali and Senegal. - Mémoire de DEA. UM1/Agro.M, 2004.

18. INSAH (INSTITUT DU SAHEL). La dévaluation du franc CFA en Afrique de I'Ouest. Quel bilan trois ans après? Etudes et recherches sahéliennes, numéro spécial. Bamako : CILSS, 1997.

19. SINGARE K, REARDON T, CAMARA Y, WANZALA $M$, TEME B, SANOGO O. Household consumption responses to the franc CFA devaluation: evidence from urban Mali. Food Policy $1999 ; 24$ : 517-34.

20. ANCEY V, et al. La hausse des prix des céréales au Niger. Volet: consommation et approvisionnement des ménages à Niamey. Club du Sahel/REDES, 1999.

21. BOCOUM I. Sécurité alimentaire et sensibilité des ménages au prix : enjeux et limites des modèles d'analyse de la demande dans les pays sahéliens. Mémoire de Master. UM1/Agro.M, 2006.

22. EGG J, MICHIELS D, BLEIN R, ALBY-FLORES V. Evaluation du Dispositif national de prévention et gestion des crises alimentaires du Niger durant la crise de 2004-2005 : rapport principal. République du Niger: Cabinet du Premier Ministre, Cellule Crises Alimentaire ; IRAM, 2006 ; (Juin).

23. TEFFTS I, PENDERS C, KELLY V, STAATZ I, YADE M, WISE V. Linkages between agricultural growth and improved child nutrition in Mali. MSU International Development Working Paper, 2000 ; (79).

24. DELPEUCH F. Evaluation du Dispositif national de prévention et gestion des crises alimentaires $d u$ Niger durant la crise de 2004-2005 : synthèse concernant les aspects nutritionnels. République du Niger : Cabinet du Premier Ministre, Cellule Crises Alimentaire ; IRAM, 2006 ; (Juin).

25. BARHOUNI M. Approvisionnement des marchés en oignon. Forum professionnel de la filière oignon en Afrique de l'Ouest. 2005.

26. BLEIN R, HAMID A, BAILLET G. Etude d'impact des Accords de Partenariat Economique (APE) sur I'économie du Niger. Iram, 2004.

27. DAVID O. Les réseaux marchands africains face à I'approvisionnement d'Abidjan: le commerce régional de l'oignon (Niger - Burkina Faso - Côte $d^{\prime}$ Ivoire). Thèse de doctorat en géographie. Université Paris X, 1999.

28. DAVID-BENZ H, BA D. L'oignon dans la vallée du fleuve Sénégal, une filière en émergence. In : Legoupil, et al., eds. Pour un développement durable de l'agriculture irriguée dans la zone 
soudano-sahélienne. Synthèse des résultats du Pôle régional de recherche sur les systèmes irrigués (PSI/CORAF), Acte du séminaire du 30/11 au 3/12/99, Dakar. 2000.

29. SECK P.A. L'approvisionnement de Dakar et les filières de légumes frais au Sénégal : Eléments de réflexion sur la définition d'une stratégie d'avenir. Thèse Doctorat. Dijon : ENSAA, 1989.
30. WADE I, DAVID-BENZ H, EGG J. Information et régulation des filières maraîchères au Sénégal. Cahiers Agricultures $2004 ; 13$ : 148-57.

31. DAVID-BENZ H, WADE I, EGG J. Market Information and Price Instability: an Insight on Vegetable Markets in Senegal. ISHS Int Conf July 2005 : 19-23 ; (Chiang Mai).
32. PELLETIER D. Etude du marché ouest-africain de I'oignon et de la pomme de terre. Vol.1, Tome 1 : Oignon. Thiès: PAEP, CECl, 2000.

33. EGG J, TALLEC F. Coordination et promotion de la qualité dans les filières "céréales sèches " au Mali.- Projet Inra-Cirad " coordination et qualité dans les filières agricoles du Sud ». Atelier $1^{\text {er }}$ et 2 juillet. Montpellier : UMR MOISA, 2004. 